\title{
Pharmacologic Trabeculectomy-Modulation of Aqueous Humor Outflow Through the Trabecular Meshwork
}

\author{
Syed Shoeb Ahmad, MS, ${ }^{1}$ Syed Zia-ur-Rahman, PhD, ${ }^{2}$ Norlina Ramli, FRCS $^{3}$ and Shuaibah Abdul Ghani, MS ${ }^{4}$ \\ 1. Specialist; 4. Consultant, Department of Ophthalmology, Queen Elizabeth Hospital, Kota Kinabalu, Malaysia; 2. Associate Professor, Department of Pharmacology, \\ Aligarh Muslim University, Aligarh, India; 3. Associate Professor, Department of Ophthalmology, University Malaya, Kuala Lumpur, Malaysia
}

\begin{abstract}
"Pharmacologic trabeculectomy" is a term used to describe the modulation of aqueous outflow by the biochemical manipulation of the trabecular meshwork. Most of the medications currently in use either reduce aqueous production or increase its outflow through the ciliary body. However, there is a new group of agents being investigated, which can increase the facility of aqueous humor outflow through the trabecular meshwork. Thus, these agents will be able to provide a more physiologic means to control aqueous humor dynamics. This review sheds light on this concept of "medical trabeculectomy."
\end{abstract}

\section{Keywords}

Aqueous humor, trabecular meshwork, glaucoma, open angle

Disclosure: Syed Shoeb Ahmad, MS, Syed Zia-ur-Rahman, PhD, Norlina Ramli, FRCS, and Shuaibah Abdul Ghani, MS, have no conflicts of interest to declare. No funding was received in the publication of this article.

Open Access: This article is published under the Creative Commons Attribution Noncommercial License, which permits any noncommercial use, distribution, adaptation, and reproduction provided the original author(s) and source are given appropriate credit.

Received: January 5, 2015 Accepted: January 26, 2015 Citation: US Ophthalmic Review, 2015;8(1):46-51 DOI: 10.17925/usor.2015.8.1.46

Correspondence: Syed Shoeb Ahmad, MS, Ophthalmology Department, Queen Elizabeth Hospital, Kota Kinabalu, 88586, Malaysia. E: syedshoebahmad@yahoo.com

"Pharmacologic or medical trabeculectomy" is a term first used in the 1970s. This describes a process by which the trabecular meshwork (TM) can be biochemically manipulated in order to reduce the outflow resistance and, thus, the intraocular pressure (IOP). The TM is now regarded as a complex organization of multiple components, which play a major role in aqueous humor $(\mathrm{AH})$ dynamics. Indeed, it is largely a function of AH dynamics to maintain IOP in a steady state. ${ }^{1}$

In cases of primary open angle glaucoma (POAG) and normal-tension glaucoma (NTG), the IOP can be controlled by either reducing the ingress of $\mathrm{AH}$ or by increasing the egress from the eye. Most of the medications in use now reduce aqueous production or the aqueous outflow through the unconventional uveoscleral pathway. According to one school of thought, decreasing the aqueous production and thus reducing aqueous outflow through the TM will progressively be detrimental to the trabecular function. This could be further accelerated by concurrent prostaglandin analog use, which directs aqueous away from the meshwork to the ciliary body. ${ }^{2}$

In order to overcome this, and to develop a new line of approach to the management of glaucoma, interest is developing on the trabecular outflow pathways, and the modulation of which, can be used to reduce IOP. In this review we take a look at the agents that have been investigated to improve the facility of $\mathrm{AH}$ outflow.

\section{Glycosaminoglycanisases}

Glycosaminoglycans (GAGs) are negatively charged particles found on the surface of TM cells, in the extracellular matrix (ECM) and the surface of cells lining the Schlemm's canal (SC). GAGs are responsible for cell matrix interactions, growth factor binding, and the sequestration and maintenance of tissue structural integrity. GAGs may induce hydration (edema) of the TM, leading to the obstruction of the trabecular structure. Agents such as testicular hyaluronidase $(T H)$, chondroitinase $A B C$, alphachymotrypsin, and cytochalasins result in IOP reduction by degrading GAGs in the ECM, disruption of actin assembly, or by preventing the polymerization of microtubules.

Hyaluronic acid (HA) was first discovered by Meyer Palmer in 1934 in bovine eyes. Soon thereafter, the enzyme hyaluronidase was discovered in pneumococci in 1936 and this was found to be identical to the spreading factor of Hoffmann and Duran-Reveals, which was obtained from testicular extracts. ${ }^{3}$ In comparison to $\mathrm{TH}$, streptomyces hyaluronidase (SH) is a more specific HA-degrading enzyme, active over a wide $\mathrm{pH}$ range, ${ }^{4-10}$ it is temperature stable, and is not inhibited by sulfated GAG polymers.

\section{Testicular Hyaluronidase}

TH was first demonstrated by Barany to reduce the outflow resistance in enucleated eyes. This agent appears to bring about structural changes 
in the juxtacanalicular tissue (JCT). It also facilitates movement of aqueous solutions through the connective tissues by acting along a pressure gradient. The initial action of TH is to reduce the interaction of mucopolysaccharide solution with the confining structures and later, when considerable degradation of the tissues has occurred, the interaction of water with the mucopolysaccharides is affected. This affects AH dynamics, leading to increased outflow. Studies have found that the TM has hyaluronidasesensitive and hyaluronidase-resistant areas, which respond differently from hyaluronidase. ${ }^{4}$

A rapid exchange of anterior chamber contents with hyaluronidase was found to produce a rapid fivefold increase in facility with stabilization of measured facility after perfusion for 3 hours or more. The changes in the facility of outflow (the so-called "washout-phenomenon") are assumed to be due to the gradual dissolution of the hyaluronidase-sensitive component of the JCT. ${ }^{5}$ Some other studies found only a moderate decrease in the HA in perfused, control eyes. They concluded that the "wash-out" effect of prolonged infusion is the result of other cellular or extracellular changes in the aqueous outflow pathway, rather than due to removal of GAGs per se. ${ }^{6}$

In another study, perfusion of TH was associated with a $50 \%$ decrease in aqueous outflow. It has been suggested that in nonprimate species there is a GAG barrier to aqueous outflow, which is sensitive to TH infusion. In human eyes, infusions of TH have given variable and inconclusive results. Infusion of 100 units of TH resulted in a moderate overall effect. However, a significant increase was observed at 30 minutes. The maximum effect was a $29 \%$ decrease in outflow resistance. However, anterior chamber injection of bovine $\mathrm{TH}$ in rabbit eyes in amounts ranging from 1.5-150 IU was found to produce persistent corneal edema, severe anterior chamber fibrin, and endothelial necrosis in majority of eyes.?

\section{Streptomyces Hyaluronidase}

Infusion of SH was found to result in a significant increase in the facility of outflow after 15 minutes. The total decrease in outflow resistance was 4-6\% for 1 unit and $50 \%$ for 10 units of $\mathrm{SH}$. This study showed $\mathrm{SH}$ is more effective than $\mathrm{TH}$ in degrading $\mathrm{HA}{ }^{6}$

\section{Alpha-chymotrypsin}

Alpha-chymotrypsin is a protease that was used to lyse lens zonular fibers during intracapsular cataract extraction. Anterior chamber perfusion of $50 \mu \mathrm{g} / \mathrm{ml}$ alpha-chymotrypsin in monkey eyes caused a marked rise in outflow facility. Structurally, the intertrabecular spaces in the angle were found to be wider than normal, openings in the uveal and corneoscleral meshwork appeared enlarged, there was ballooning of the juxtacanalicular TM and innerwall of the SC into the canal, and also splitting of the innerwall of the canal. ${ }^{8}$ Bill reported that a 30 -minute perfusion of $50 \mathrm{U} / \mathrm{ml}$ alpha-chymotrypsin lead to a marked rise in outflow conductance, which was well maintained over 2 hours. No side effects were noted during the experiment or for a few weeks subsequently. ${ }^{9}$ However, Melena noted an increase in the $\mathrm{AH}$ protein levels attributed to the breakdown of the blood aqueous barrier leading to ocular hypertension in rabbits. ${ }^{10}$

\section{Calcium Chelators}

Calcium ions are required for cell adhesion and permeability in many systems. Thus, removal of calcium ions ( $\mathrm{Ca++}$ ) from the $\mathrm{AH}$ can alter the
Table 1: Classification of Aqueous Outflow Modifying Agents

\begin{tabular}{lll} 
Enzymatic Agents & $\begin{array}{l}\text { Cytoskeletal and Cell } \\
\text { Adhesive Agents }\end{array}$ & $\begin{array}{l}\text { Ethacrynic Acid and } \\
\text { Sulfhydryl-reactive Agents }\end{array}$ \\
\hline Glycosaminoglycanisases & Calcium chelators & lodoacetamide \\
\hline Alpha-chymotrypsin & Cytochalasins & N-methyl malemide \\
\hline & Colchicine & Ethacrynic acid \\
\hline H7 & \\
\hline & Latrunculins & \\
Rho-associated coiled \\
coil-forming protein \\
kinase inhibitors
\end{tabular}

biochemical properties of the TM. This would affect $\mathrm{AH}$ dynamics and "might be a procedure of interest in the treatment of glaucoma."11

Calcium-free fluid containing sodium ethylene diamine tetracetic acid (Na2EDTA) or (ethylene glycol-bis- $\beta$-amino-ethyl ether)-N-N^-tetracetic acid (EGTA) have been used as a chelating agents to bind $\mathrm{Ca}++$ entering the anterior chamber through the $\mathrm{AH}$ or by diffusion from the tissues. ${ }^{11}$

Experiments have shown that infusion of Na2EDTA led to a marked rise in gross outflow facility at $4 \mathrm{mM}$ as well as at $6 \mathrm{mM}$. The outflow facility was found to increase 20 minutes after the start of the perfusion and reached a maximum at 40-50 minutes. The adverse events noted were pupillary dilatation and corneal cloudiness, which remained for 1-2 weeks before subsequently subsiding. Similarly, 80-90 minutes of EGTA infusion also caused a marked increase in gross outflow facility, which was however partly reversible. These effects have been attributed to the binding of Na2EDTA to $\mathrm{Ca}++$ and $\mathrm{Mg}++$, while EGTA is a rather specific $\mathrm{Ca}++$ chelator. ${ }^{11}$

The decrease in outflow resistance has been attributed to: loss of cell processes from trabecular cells, damaged trabecular cores, distention of the cribriform meshwork with loss of ECM, ballooning of the IW of the SC into the SC, and ruptures in the IW. ${ }^{12}$

Hamanaka reported the AC perfusion of $0.5 \mathrm{mM}$ of Na2EDTA in monkey eyes to be effective in increasing the facility of aqueous outflow within 60 minutes. However, after interruption of perfusion there was rapid reduction in outflow facility, which is presumed to be due to occlusion of the ruptures in the SC by thrombocytes. ${ }^{13}$

The commercial application of calcium chelators in glaucoma management is yet to be seen. Na2EDTA suffers from the disadvantage of not being able to penetrate the corneal epithelium. Thus, it has to be infused directly into the AC or possibly by the use of iontophoresis. ${ }^{11}$

\section{Cytochalasins}

Cytochalasins are fungal metabolites that were found to interfere with the polymerization process by which globular cytoplasmic actin aggregates into microfilaments of actin. Anterior chamber infusion of cytochalasin-B and -D in monkey eyes was found to cause distention of the cribriform meshwork, separation of its cells, and ruptures in the IW endothelium, leading to washout of the extracellular material. This also lead to a sixfold increase 
in conventional outflow facility. These agents also cause disruption of actin filaments. Studies have found that a continuous exposure of cytochalasin B in a concentration of more than $15 \mu \mathrm{g} / \mathrm{ml}$ for 30 minutes causes sufficient disruption of the endothelial lining leading to extensive washout of the extracellular material in the trabecular meshwork. ${ }^{14}$

Johnson reported around $40 \%$ increase in the facility of aqueous outflow after infusion of 0.6 and $1.1 \mathrm{mg} / \mathrm{ml}$ of cytochalasin $\mathrm{D}$. The onset of effect started within 40 minutes, peaked at 2 and 6 hours, and lasted at least 6-14 hours. ${ }^{15}$ Cytochalasins also act by capping the filaments and preventing their elongation, thereby affecting a wide variety of actindependent cellular events including cell morphogenesis, motility, and endocytosis. Svedbergh, in his study, also reported a consistently large increase in outflow facility with $5 \mu$ g of intracameral cytochalasin B in cynomolgus monkey eyes. ${ }^{16}$

Cytochalasin B increases the hydraulic conductivity of cultured monolayers of human TM cells, accompanied by a retraction of the cells and a widening of the intercellular spaces. It is also reported to cause a disruption of endothelial lining and a washout of extracellular material in the TM tissue. These actions contribute to the increased AH outflow. ${ }^{17,18}$

Cytochalasins bind to the growing end of the microfilaments, capping them and prevent the polymerization of actin monomers into F-actin containing microfilaments. ${ }^{19}$ Actin filaments are normally in a state of polymerization and depolymerization. Cytochalasin interferes with the polymerization, leading to shifting of the equilibrium towards eventual depolymerization of the microfilaments. Cytochalasin D in humans has been reported to produce changes that were similar to those seen in primates with cytochalasin B. Cells in the uveal and corneoscleral meshwork remained intact, unlike findings in a study of monkeys where separation, degeneration, and disappearance of meshwork cells in this region was observed. The facility of outflow returns back to baseline after a few hours as the IW endothelium cells get replaced, with plugging of the breaks with platelets and synthesis of the lost extracellular matrix components. ${ }^{15}$

\section{Colchicine}

Colchicine is a plant alkaloid that binds specifically with tubulin on topical or intravitreal injection. Thus, it prevents polymerization of microtubules in various locations in the eye.12,20 A topical application of 50 or $100 \mu g$ of colchicine was found to produce significant lowering of IOP after 24 hours. The mechanism of action of colchicine is through an increase in the rate of aqueous outflow. However, despite continuous administration of colchicine the IOP returns to baseline. Colchicine administration is also associated with significant ocular toxicity to the cornea and other anterior segment structures. Subsequently, IOP becomes elevated due to obstruction or disorganization of the outflow pathways. Lumilcolchicine is an inactive isomer with insignificant affinity for tubulin. This isomer does not affect aqueous outflow, thus the mode of action of colchicine is through its interaction with tubulin and cytoskeletal elements in the trabecular meshwork.12

A study by Williams on albino rabbits showed that topically administered colchicine can also decrease the $\mathrm{AH}$ production in a dose-dependent manner. The mean rate of $\mathrm{AH}$ formation in control eyes was $3.68 \pm 0.09$ $\mu \mathrm{l} / \mathrm{min}(n=16)$. While, it decreased to $1.8 \mu \mathrm{l} / \mathrm{min}$ in eyes treated with $40 \mu \mathrm{g}$ colchicine. In eyes treated with $10 \mu g / e y e$, the gross outflow facility was found to be $0.49 \pm 0.03 \mu \mathrm{l} / \mathrm{minute} / \mathrm{mmHg}$, which was significantly greater than that of the contralateral control eyes $(0.39 \pm 0.03 \mu \mathrm{l} / \mathrm{minute} / \mathrm{mmHg}){ }^{21}$

\section{H7}

$\mathrm{H} 7$ (1-(5-isoquinolinyl-sulfonyl)-2-methylpiperazine) is a broad-spectrum serine-threonine kinase inhibitor. It has been found to reversibly increase outflow facility across the TM and, consequently, reduce the IOP.22 It blocks the phosphorylation activity of diverse serine-threonine kinases including protein kinase $\mathrm{C}$, myosin light chain kinase (MLCK), and Rho kinase. It also reduces the actinomyosin-driven contractility and leads to deterioration of the actin microfilament system. ${ }^{23,24}$

Adhesions between cells and between cells and the ECM in the TM contribute to the geometry and flow resistance within the TM. These adhesions include complex transmembrane interactions between the external cell surface and the actin-based cytoskeleton. This is mediated by specific adhesion receptors (integrins and cadherins) and a variety of submembrane anchor proteins (e.g. vinculin and catenins), which link the microfilaments to the membrane in these sites. ${ }^{25}$

$\mathrm{H} 7$ treatment has been found to weaken cell-cell or cell-ECM junctions in cultured cells. H7-treated cells appear to round or thicken towards the centre in a dose- and time-dependent manner. $\mathrm{H} 7$ can destabilize the overall architecture of the TM and SC by inducing changes in cell shape and cellular attachments. This opens the flow pathways and leads to formation of new flow routes, thereby reducing flow resistance and IOP. A generalized relaxation and apparent expansion of the TM and SC occurs on treatment with $\mathrm{H7}$. It also causes a secondary actin degradation that is, however, considered less physiologically relevant. ${ }^{22}$

In a study by Liu, treatments with $100 \mu \mathrm{M}$ of $\mathrm{H} 7$ led to a mild effect of cell rounding and apparent thickening towards the centre of cells, within 1 hour of exposure. After exposure to $300 \mu \mathrm{M}$ of $\mathrm{H} 7$ for 1 hour the cells became more refractile towards the centre. After 24 hours of exposure to $100 \mu \mathrm{M}$ or $300 \mu \mathrm{M}$ of $\mathrm{H7}$, nearly all treated cells showed these changes. Also, actin-containing bundles were found to deteriorate rapidly after 30 minutes of exposure to $\mathrm{H} 7$, especially at higher doses. With longer exposures (2 hours), the stress fiber network was obliterated leaving only a diffuse mesh of actin filaments, mostly at the cell periphery. ${ }^{22}$

Side effects noted in the above study include a transiently increased protein concentration in the anterior chamber on treatment with $400 \mathrm{mM}$ of $\mathrm{H7}$. There was also an increased entry of intravenous fluorescein into the $\mathrm{AH}$ and cornea. There was an increase in the corneal thickness, with the corneal endothelial cell borders found to be indistinct on specular microscopy.

Tian noted a significant IOP reduction during 1 to 6 hours after topical instillation of $\mathrm{H} 7$ at a concentration of $400 \mathrm{mmol} / \mathrm{I}$ with a maximal decrease of $3.25 \mathrm{mmHg}$ at hour 2 compared with that in the contralateral control eyes. The authors did not note any anterior chamber or lens abnormalities on topical or intracameral administration of $\mathrm{H} 7 .{ }^{25}$

\section{Latrunculins}

Latrunculins are macrolides isolated from the marine sponge Negombata magnifica (formerly Latrunculia magnifica). Macrolides are compounds 
whose activity stems from the presence of a macrolide ring, a large macrocyclic lactone ring, to which one or more deoxy sugars might be attached. The two common latrunculins are latrunculin-A (LAT-A) and latrunculin-B (LAT-B). They differ structurally in the macrolide part, which could play a part in permeation into cells. LAT-A has a diene moiety, while LAT-B incorporates a monoene moiety. LAT-B, unlike LAT-A, is found to be slowly inactivated by an unknown serum component in cell culture medium. Thus, 48 hours after exposure to LAT-B, the cells completely recover, exhibiting a well-developed system of stress fibers. LAT-B also has a milder effect on corneal endothelial permeability compared with LAT-A. Unlike cytochalasin B and ethacrynic acid (ECA), LAT-B does not induce cell-cell separations in the live monkey eye, although it disrupts human TM cell-cell junctions in culture. . $^{26-28}$

LAT-A is a specific actin-disrupting agent that disassembles and disrupts polymeric actin filaments (F-actin) by sequestering monomeric actin (G-actin). It also causes cell separation and cell loss. Topical or intracameral LAT-A reversibly and in a dose- and time-dependent manner increases the outflow facility and lowers the IOP. LAT-A directly binds with G-actin and disrupts the actin microfilament network and subsequently interferes with cell contractility and adhesion. (H7 affects contractility, with secondary deterioration of actin microfilamentary system). LAT-A may also work by affecting other outflow-relevant cells in the chamber angle (e.g. collector channels, episcleral veins), as well as through other uncertain physiologic consequences. ${ }^{29}$

LAT-B is about 10 times more potent than LAT-A in increasing the facility of outflow, but has fewer effects on corneal endothelial permeability. Thus, LAT-B is assumed to be "gentler" to the ocular tissues. On perfusion of porcine eyes with LAT-B and LAT-A no signs of cellular toxicity were observed. In living monkey eyes, LAT-A was found to reduce IOP after 6 hours of administration. Contrarily, LAT-B brought the IOP down within 1 hour. A study by Ethier showed that perfusion with LAT-B in a concentration of $1 \mu \mathrm{M}$ had a dramatic improvement in outflow facility in enucleated human eyes. This was equivalent in magnitude to that seen in enucleated porcine eyes, but less than that observed in living monkey eyes. ${ }^{30}$ LAT-B produces cytoskeletal disorganization in TM cells and IW cells of SC producing a substantial expansion of the space between the IW of SC and the trabecular collagen beams. ${ }^{31}$ Under baseline conditions, the IW and JCT cells are in a contracted state. A reduction in their contractility expands the tissues, altering the routes and extent of fluid flow.

Okka and colleagues studied the effects of single versus multiple doses of $0.005 / 0.01 \%$ topical LAT-B on the outflow facility, IOP, and/or central corneal thickness in normotensive monkey eyes. Fifteen treatments of $0.01 \%$ LAT-B were found to significantly increase the outflow facility in monkey eyes. The effect of the drug was found to last for more than 16 hours and no corneal toxicity was noted. ${ }^{2}$ Similarly, Peterson noted a dose-dependent two to threefold increase in outflow facility after a topical $21 \mu$ instillation of LAT-A. ${ }^{29}$

LAT-A and LAT-B produce multiple small, brightly refractile, granule-like spots, or pseudoguttata on the corneal endothelium. ${ }^{31}$ LAT-A and -B have been found to increase protein concentration in the anterior chamber, induce cell shape changes and increased permeability in the corneal endothelium, and lead to overall corneal thickening. These changes are usually mild and reversible. However, Peterson and colleagues studied LAT-A topical/perfusion in monkeys and did not notice any corneal changes on biomicroscopy. ${ }^{28}$

\section{Rho-associated Coiled Coil-forming Protein Kinase Inhibitors}

The Rho-associated coiled coil-forming protein kinase (ROCK) is a kinase that regulates the movement and shape of cells by its action on the cytoskeleton. It is a downstream effecter of Rho in the Rho-dependent signal transduction pathway. ROCKs also lead to an abnormal accumulation of ECM (ECM hypothesis) and changes in contractile activity and cell adhesive interactions of the cells of aqueous outflow pathway (contractility hypothesis) leading to an increased resistance to drainage of $\mathrm{AH}$ through the conventional pathway. Specific inhibitors of ROCK modulate changes in the actin cytoskeleton and affect cellular motility at the level of trabecular meshwork, SC, and ciliary muscle, augmenting aqueous outflow. ${ }^{32}$

ROCK inhibitors are a potential new class of ocular hypotensive drugs. While more than a dozen ROCK inhibitors are known, only a few have been studied for their potential role as antiglaucoma medications. These agents include Y-27632, AR-12286, SNJ-1656/Y 39983/RKI 983, K 115, and INS 117548. However, as of 2014, only some of them were undergoing human trials, the development of others having been discontinued. These agents include: AMA 0076, AR-13324, K-115, and PG-324. ${ }^{33}$

AMA 0076 is a novel, locally acting Rho kinase inhibitor. It is structurally related to Y-27632. Previous studies have reported Y-27632 to produce Rho/ROCK dependent F-actin reorganization, impairment of focal adhesion formation and decreased MLC phosphorylation in TM and SC endothelial cells. This results in an increase in the facility of aqueous outflow. ${ }^{34,35}$ AMA 0076, developed by Amakem Therapeutics, Diepenbeek, Belgium, involves a soft drug approach, involving predictable metabolic inactivation of an active compound to a nontoxic metabolite, thus improving tolerability. compared with latanoprost and bimatoprost, this compound was found to be more potent in preventing IOP spikes in an acute hypertensive rabbit model. No significant side effects, especially hyperemia were noted. ${ }^{36,37}$

AR13324 inhibits both Rho-kinase and norepinephrine transporter to increase trabecular outflow and decrease aqueous production as well. Topical AR13324 $0.02 \%$ and latanoprost in once-daily dosing were found to be equally effective in controlling IOP. The only side effect noted was mild to moderate conjunctival hyperemia. ${ }^{38}$

The AR-13324-CS202 Study Group performed a double-masked study comparing AR-13324-0.01 \% and -0.02 \% with latanoprost. AR-13324 $0.02 \%$ was found to be less effective than latanoprost by approximately $1 \mathrm{mmHg}$ in patients with unmedicated IOPs of 22 to $35 \mathrm{mmHg}$. The major adverse event was ocular hyperemia, which was more common for both concentrations of AR-13324 than for latanoprost. ${ }^{39}$

Aerie Pharmaceuticals (Bedminster, New Jersey, US) has developed an agent named PG324 that combines AR13324 and latanoprost. The phase II trials aim to detect the effectiveness of this drug. This is regarded as the first antiglaucoma medication with three distinct mechanisms.

$\mathrm{K}-115$ is a highly selective and potent isoquiline sulfonamide compound. It is undergoing phase II evaluation. Preclinical testing of the compound in 
monkey eyes showed a 2.3-4 mmHg decrease in the IOP compared with a $2.5 \mathrm{mmHg}$ decrease with $0.005 \%$ latanoprost. ${ }^{34}$

\section{Iodoacetamide}

Metabolic inhibitors have been used in the past to modify AH outflow. Experiments by Barany on iodoacetic acid injection into the anterior chamber of calf eyes showed an increase in the facility of outflow by affecting the hyaluronidase-insensitive component of outflow resistance. ${ }^{40}$ Studies also show that iodoacetate can have specific effects on passive cellular membrane permeability, which are independent of their metabolic effects. It is assumed that membrane sulfhydryl groups in the trabecular endothelium may be directly involved in aqueous outflow dynamics and are modified by iodoacetamide.

Studies by Epstein showed that iodoacetamide causes an increase in the facility of aqueous outflow in a dose-response manner in calf and monkey eyes. This could be attributed to the inhibitory effect on glycolysis and energy production in the trabecular meshwork. However, TM excised from perfused as well as fresh, nonperfused eyes demonstrated a similar rate of glycolysis. A 15 and $30 \mu \mathrm{mol}$ infusion of iodoacetamide significantly increased the facility of aqueous outflow. A single dose of $30 \mu \mathrm{mol}$ of iodoacetamide was effective but not as much as those eyes that had been treated previously with multiple lower doses of iodoacetamide. ${ }^{41}$

\section{N-methylmalemide}

Sulfhydryl (-SH) groups are one of the most prevalent and reactive ligands in biological tissues and are present in most cellular proteins. -SH also affect the aqueous outflow, presumably by an inhibitory action on energy production in the trabecular meshwork. But, more likely, there is a direct effect on aqueous outflow by the cellular -SH groups and related membrane activities. The state of cell membrane protein sulfhydryls may also affect the cellular or intercellular permeability to fluid flow in the aqueous outflow channels. Epstein and colleagues used N-ethylmaleimide (NEM), a specific -SH agent to study its effect on aqueous outflow. They found that a dosage of $4.7 \mathrm{mM}$ or greater of NEM produced a significant increase in the facility of outflow in the calf and monkey eyes. ${ }^{42}$

\section{Ethacrynic Acid}

Ethacrynic acid (ECA) is an -SH-reactive agent that has been used as a systemic diuretic. It causes a dramatic reversible change in the cell shape in different cell lines including human trabecular, calf, and bovine pulmonary artery endothelial cells associated with changes in actin, $\alpha$-actinin, vimentin, and vinculin. These shape changes are preceded by disruption and subsequent loss of $\beta$-tubulin staining. ECA is found to bind with tubulin and may affect tubulin metabolism directly. ${ }^{43} \mathrm{AC}$ perfusion with this agent lead to breaks in IW endothelial lining of the SC and loss of cell-cell attachments between the IW and the subendothelial trabecular cells and between trabecular cells themselves. ${ }^{44}$

Ethier and colleagues, in their studies on living monkey eyes, found a $63 \%$ increase in the outflow facility after intracameral perfusion with ECA. They also noted increased platelet adhesion to the IW of SC, decreased I pore density, and a tendency of increasing density of large B pores..$^{45}$ Conversely, Epstein reported only a $20 \%$ increase in the net facility shortly after a bolus injection of $10 \mu \mathrm{l}$ of $2.5 \mathrm{mM}$ ECA into the anterior chamber. ${ }^{46}$ However, Croft reported a $71 \%$ increase in the outflow facility 1 hour after drug infusion. ${ }^{43}$ Melamed and colleagues injected intracameral ECA in patients with advanced OAG. They reported a drop in IOP ranging from 9-31 mm Hg after 5-15 $\mu$ l ECA injection. The effect was noted 3-24 hours after treatment and lasted for 3 days. The IOP gradually returned to pretreatment values 1 week later. ${ }^{47}$

No clinically apparent side effects have been reported in the anterior segments of cynomolgus/rhesus monkeys after intracameral ECA injections. Since these were young, healthy monkeys, it remains to be seen if the eyes of elderly glaucoma patients would behave similarly. ${ }^{43}$ However, Melamed in his study of intracameral ECA in OAG patients did not observe any adverse effects on the cornea or anterior chambers. This led him to suggest that ECA could be used as a new class of drugs to treat OAG. ${ }^{47}$

Some new drugs are being developed by modifying phenoxyacetic acid and acrylol moieities. These compounds were evaluated on the basis of effects produced on TM cell morphology, actin cytoskeleton, focal adhesions, and transcellular fluid flow. Based on these evaluations a compound SA9000 was found to produce a maximal reduction in IOP with minimal side effects compared with ECA, on either intracameral injection or topical instillation. ${ }^{48}$

\section{Conclusion}

Pharmacologic management of glaucoma has been successful in controlling IOP by reducing aqueous inflow through suppression of aqueous production or by increasing the aqueous outflow primarily via the unconventional uveal pathway. However, recently a new frontier in pharmacologic modulation of the facility of aqueous outflow through the TM has opened up. This concept of pharmacologic trabeculectomy raises the possibility of a more physiologic control of the aqueous outflow and achieving better control of the IOP. The present review sheds some light on these exciting developments that may be another weapon in our armamentarium to manage glaucoma.
1. Ahmad SS, Ghani SA, Singh D, et al., The dynamics of aqueous humor outflow: A major review, US Ophthalmic Review 2014:7:137-42.

2. Okka M, Tian B, Kaufman PL, Effects of latrunculin B on outflow facility, intraocular pressure, corneal thickness, and miotic and accommodative responses to pilocarpine in monkeys, Trans Am Ophthalmol Soc, 2004;102:251-9.

3. Mascati NT, Experiences with hyaluronidas, Indian I Ophthalmol, 1953:1:23-8.

4. Gum GG, Samuelson DA, Gelatt KN, Effect of hyaluronidase on aqueous outflow resistance in normotensive and glaucomatous eyes of dogs, Am J Vet Res, 1992:53:767-70.

5. Van Buskirk EM, Brett J, The canine eye: in vitro studies of the intraocular pressure and facility of aqueous outflow, Invest Ophthalmol Vis Sci, 1978;17:373-7.

6. Knepper PA, Farbman Al, Telser AG, Exogenous hyaluronidases and degradation of hyaluronic acid in the rabbit eye, Invest
Ophthalmol Vis Sci, 1984;25:286-93

7. Jumper JM, McCauley MB, Duncan KG, et al., Corneal toxicity of intraocular hyaluronidase, J Ocul Pharmacol Ther. 2002;18:89-97.

8. Hamanaka T, Bill A, Effects of alpha-chymotrypsin on the outflow routes for aqueous humor, Exp Eye Res, 1988;46:323-41.

9. Bill A, Effects of Na2 EDTA and alpha-chymotrypsin on aqueous humor outflow conductance in monkey eyes, Ups I Med SCi, 1980;85:311-9.

10. Melena J, Santafé J, Segarra-Doménech J, et al., Aqueous humor dynamics in alpha-chymotrypsin-induced ocular hypertensive rabbits, J Ocul Pharmacol Ther, 1999:15:19-27.

11. Bill A, Lutjen-Drecoll E, Svedbergh B, Effects of intracameral Na2EDTA and EGTA on aqueous outflow routes on aqueous outflow routes in the monkey eye, Invest Ophthalmol Vis Sci, 1980;19:492-504.

12. Allingham RR, Medical trabeculectomy. In: Zimmerman TJ,
Kooner KS (eds), Textbook of Ocular Pharmacology, Philadelphia: Lippincott Raven Publishers, 1997:341-45.

13. Hamanaka T, Bill A, Morpholgical and functional effects of sodium EDTA on the outflow routes for aqueous humor in monkeys, Exp Eye Res, 1987;44:171-90.

14. Johnstone M, Tanner D, Chau B, et al., Concentration-dependent morphologic effects of cytochalasin B in the aqueous outflow system, Invest Ophthalmol Vis Sci, 1980;19:835-41.

15. Johnson $\mathrm{DH}$, The effect of cytochalasin $\mathrm{D}$ on outflow facility and the trabecular meshwork of the human eye in perfusion organ culture, Invest Ophthalmol Vis Sci, 1997:38:2790-9.

16. Svedbergh $B$, Lütien-Drecoll $E$, Ober M, et al., Cytochalasin B-induced structural changes in the anterior ocular segment of the cynomolgus monkey, Invest Ophthalmol Vis Sci, 1978;17:718-34.

17. Perkins TW, Alvarado JA, Polansky JR, et al., trabecular meshwork cells grown on filters, Invest Ophthalmol Vis Sci, 
1988;29:1836-46.

18. Robinson JC, Kaufman PL, Cytochalasin B potentiates epinephrine's outflow facility-increasing effect, Invest ophthalmol Vis Sci, 1991;32:1614-18.

19. Brown SS, Spudich JA, Cytochalasin inhibits the rate of elongation of actin filament fragments, I Cell Biol, 1979;83:657-62

20. Ritch R, Mulberg A, Rosen C, et al., The effect of colchicine on aqueous humor dynamics, Exp Eye Res, 1981;32:143-50.

21. Williams RN, Cole DF, Bhattacherjee $P$, The effect of colchicine on the rate of formation of aqueous humour and the gross outflow facility in the albino rabbit, Exp Eye Res, 1983:36:711-7.

22. Liu X, Cai S, Glasser A, et al., Effect of H-7 on cultured human trabecular meshwork cells, Mol Vis, 2001. 27;7:145-53.

23. Tian B, Gabelt BT, Geiger B, et al., Combined effects of $\mathrm{H}-7$ and cytochalasin B on outflow facility in monkeys, Exp Eye Res, 1999;68:649-55.

24. Sabanay I, Tian B, Gabelt BT, et al., Functional and structural reversibility of $\mathrm{H7}$ effects on the conventional aqueous outflow pathways in monkeys, Exp Eye Res, 2004:78:137-50.

25. Tian B, Kaufman PL, Volberg T, et al., H-7 disrupts the actin cytoskeleton and increases outflow facility, Arch Ophthalmol, 1998;116:633-43.

26. Sabanay I, Tian B, Gabelt BT, et al., Latrunculin B effects on trabecular meshwork and corneal endothelial morphology in monkeys, Exp Eye Res, 2006;82:236-46.

27. Peterson JA, Tian B, Geiger B, et al., Effect of latrunculin-B on outflow facility in monkeys, Exp Eye Res, 2000:70:307-13.

28. Cai S, Liu X, Glasser A, et al., Effect of latrunculin-A on morphology and actin-associated adhesions of cultured human trabecular meshwork cells, Mol Vis, 2000;6:132-43.
29. Peterson JA, Tian B, Bershadsky AD, et al., Latrunculin-A increases outflow facility in the monkey, Invest Ophthalmol Vis SCi, 1999:40:931-41.

30. Ethier CR, Read AT, Chan DW, Effects of latrunculin-B on outflow facility and trabecular meshwork structure in human eyes, Invest Ophthalmol Vis Sci, 2006;47:1991-8.

31. Peterson JA, Tian B, MCLaren JW, et al., Latrunculins' effects on intraocular pressure, aqueous humor flow, and corneal endothelium, Invest Ophthalmol Vis Sci, 2000;41:1749-58.

32. Chen J, Runyan SA, Robinson MR, Novel ocular antihypertensive compounds in clinical trials, Clin Ophthalmol, 2011;5:667-77.

33. Wang SK, Chang RT, An emerging treatment option for glaucoma: Rho kinase inhibitors, Clin Ophthalmol, 2014;8:883-90.

34. Kameda T, Inoue T, Inatani M, et al., The effect of Rho-associated protein kinase inhibitor on monkey Schlemm's canal endothelia cells, Invest Ophthalmol Vis Sci, 2012;53:3092-103.

35. Tian B, Kaufman PL, Effects of the Rho kinase inhibitor Y-27632 and the phosphatase inhibitor calyculin A on outflow facility in monkeys, Exp Eye Res, 2005;80:215-25.

36. Boland, S, Defert O, Alen J. et al., 3-2-(Aminomethyl)-5-(pyridin4 -yl)carbamoylphenyl benzoates as soft ROCK inhibitors, Bioorg Med Chem Lett, 2013;23:6442-6.

37. Van de Velde S, Van Bergen T, Sijnave D, et al., AMA0076, a nove locally acting Rho kinase inhibitor, potently lowers intraocular pressure in New Zealand white rabbits with minimal hyperemia, Invest Ophthalmol Vis Sci, 2014;55:1006-16.

38. Wang RE, Williamson JE, Kopczynski C, et al., Effect of $0.04 \%$ AR-13324, a ROCK, and norepinephrine transporter inhibitor, on aqueous humor dynamics in normotensive monkey eyes, J Glaucoma, 2015:24:51-4.

39. Bacharach J, Dubiner HB, Levy B, et al., Double-masked
Randomized, Dose-Response Study of AR-13324 versus Latanoprost in Patients with Elevated Intraocular Pressure, Ophthalmology, 2015:122:302-7.

40. Barany $\mathrm{H}$, In vitro studies of the resistance to flow through the angle of the anterior chamber, Acta Soc Med Ups, 1954;59:260-76.

41. Epstein DL, Hashimoto JM, Anderson PJ, et al., Effect of iodoacetamide perfusion on outflow facility and metabolism of the trabecular meshwork, Invest Ophthalmol Vis SCl, 1981;20:625-31.

42. Epstein DL, Patterson MM, Rivers SC, et al., $\mathrm{N}$-ethylmaleimide increases the facility of aqueous outflow of excised monkey and calf eyes, Invest Ophthalmol Vis Sci, 1982;22:752-6.

43. Croft MA, Hubbard WC, Kaufman PL, Effect of ethacrynic acid on aqueous outflow dynamics in monkeys, Invest Ophthalmol Vis SCi, 1994;35:1167-75.

44. Erickson-Lamy K, Schroeder A, Epstein DL, Ethacrynic acid induces reversible shape and cytoskeletal changes in cultured cells, Invest Ophthalmol Vis Sci, 1992;33:2631-40.

45. Ethier CR, Croft MA, Coloma FM, et al., Ethacrynic acid effects on inner wall pores in living monkeys, Invest Ophthalmol Vis SCi, 1999:40:1382-91.

46. Epstein DL, Freddo TF, Bassett-Chu S, et al., Influence of ethacrynic acid on outflow facility in the monkey and calf eye, Invest Ophthalmol Vis Sci, 1987;28:2067-75.

47. Melamed S, Kotas-Neumann R, Barak A, et al., The effect of intracamerally injected ethacrynic acid on intraocular pressure in patients with glaucoma, Am J Ophthalmol, 1992;113:508-12.

48. Rao PV, Shimazaki A, Ichikawa M, et al.. Effects of novel ethacrynic acid derivatives on human trabecular meshwork cell shape, actin cytoskeletal organization, and transcellular fluid flow, Biol Pharm Bull, 2005;28:2189-96. 